\title{
Hypofractionated radiotherapy part of multimodality treatment in multicentric glioblastoma multiforme: A case report
}

\author{
Vanessa Moldoveanu', Rodica Anghel ${ }^{1,2}$, Alina Stoica $^{3}$, Mihai-Teodor Georgescu ${ }^{1,2}$ \\ 1"Carol Davila” University of Medicine and Pharmacy, Bucharest, Romania \\ 2"Prof. Dr. Alexandru Trestioreanu" Institute of Oncology, Bucharest, Romania \\ ${ }^{3}$ OncoFort Hospital, Bucharest, Romania
}

\begin{abstract}
Objective. Multicentric glioblastoma multiforme (mGBM) is a rare entity with worse prognostic than single lesion peer and impose diagnostic and therapeutic challenges.

Case presentation. We report the case of a 63-year-old man presented with left-sided palsy, paresthesia, dizziness and dysarthria. The brain magnetic resonance imaging (MRI) showed two supratentorial contrast-enhancing lesions: first in the right thalamic region and second in the left temporal lobe. Stereotactic biopsy of the right tumor was performed and the pathology report revealed glioblastoma multiforme (GBM) grade IV. The patient underwent radiotherapy with concomitant Temozolomide (TMZ). After MRI evaluation, which described left temporal tumor progression, the patient received 3 cycles of $T M Z$ and Irinotecan, followed by hypofractionated reirradiation and maintenance TMZ. Disease stabilisation was achieved and monitored for the last 11 months without neurological status worsening due to multiples therapies.

Conclusion. Prolonged tumoral control and survival was obtained in a multicentric GBM patient, similar to single lesion counterpart, by using a multimodal personalized approach.
\end{abstract}

Keywords: multiple gliomas, multicentric, glioblastoma multiforme, hypofractionated radiotherapy, reirradiation

\section{INTRODUCTION}

Multicentric gliomas are unusual tumors with an incidence that ranges widely between $2-16.2 \%$ among different reporting (1-5), with glioblastoma being the most common histological type $(2,6)$. The features that characterise multicentricity, as described by Batzdorf and Malamud (1), implies a total separation of the lesions in different lobes or hemispheres without a clear growth or dissemination pathway as in multifocal cases (via white matter tract, cerebrospinal fluid routes, blood vessels or local metastasis). MRI increasing usage is able to better differentiate the two categories (3).

Although local treatments, like surgery and radiotherapy, vary from stereotactic biopsy to multi- ple craniotomies (7) and from external beam delivery to brachytherapy (8), associated or not to systemic Temozolomide, the management of mGBM remains controversial and patient prognosis is less favourable $(4,9,10)$.

Here we present the case of synchronous multicentric glioblastoma treated with a multimodality approach achieving important disease stabilization beyond progression and tolerable toxicity.

\section{CASE PRESENTATION}

A 63-year-old man, with no prior medical history presented with left-sided motor and sensory impairment and dizziness. Initial neurological examination revealed a conscious, oriented patient with 


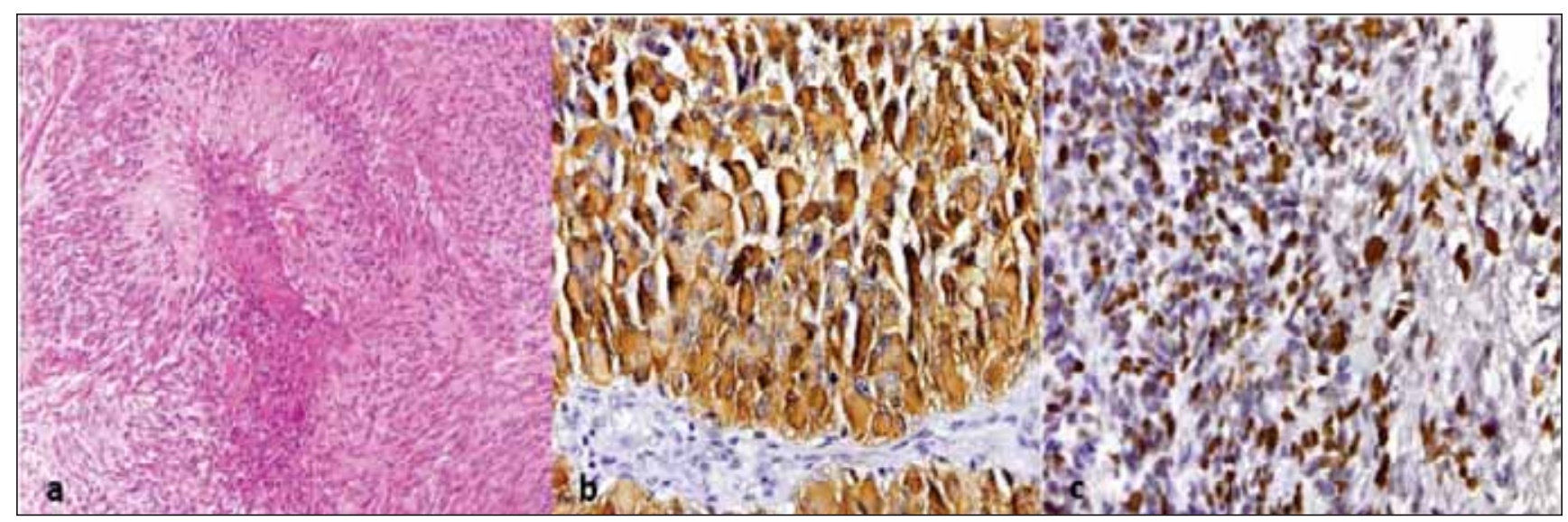

FIGURE 1. GBM histology: Hematoxylin and eosin (H\&E) staining showing necrosis and hyperchromatic nuclei (a). GBM immunohistochemistry: GFAP positive in tumor cell (b) and high Ki67 proliferation index (c).

left spastic hemiparesis with 3/5 Medical Research Council (MRC) (11) motor strength, left-right tendons reflexes asymmetry, left superficial hemihypesthesia and dysarthria.

Front cranial computed tomography (CT) identified a $48 / 36 \mathrm{~mm}$ right thalamic mass nearby the corresponding lateral ventricle and another $21 \mathrm{~mm}$ left temporal one, both suggesting cerebral metastases. No primary tumor was found on subsequent body scans. Further, brain MRI showed two distinct supratentorial contrast-enhancing lesions: first of 52/40 $\mathrm{mm}$ in the right thalamic region, extended to right mesencephalon with a $5 \mathrm{~mm}$ midline shift and right lateral ventricle displacement, and the second of 28/26 mm in the left temporal lobe in close contact with the ipsilateral ventricle.

Stereotactic biopsy of the right tumor was performed with a frozen section diagnosis of malignant glioma. The histopathology and immunohistochemical staining (Figure 1) revealed glioblastoma multiforme (GBM) grade IV, glial fibrillary acidic protein (GFAP) positive, isocitrate dehydrogenase-1 (IDH-1) negative, $\mathrm{O}^{6}$-methylguanine-DNA methyltransferase (MGMT) positive with Ki67 proliferation index of $20 \%$. Fluorescence in situ hybridization (FISH) analysis detected $1 \mathrm{p}$ chromosome deletion and absence of $19 \mathrm{q}$ co-deletion.

Four weeks following biopsy, the patient presented with an Eastern Cooperative Oncology Group (ECOG) (12) performance status of 3 and initiated radiotherapy combined with oral alkylating agent Temozolomide $75 \mathrm{mg} / \mathrm{m}^{2}$ daily according to Stupp et al.(13) protocol.

A $3 \mathrm{~mm}$ contrast-enhanced CT study (Figure 2) was performed with the patient in an easily reproducible supine position and fixed with a thermoplastic mask. In order to improve tumoral detection, all CT simulation images were fused with previous MRI set. Target volumes were defined as follows: gross tumor volume (GTV) as the gadolin-

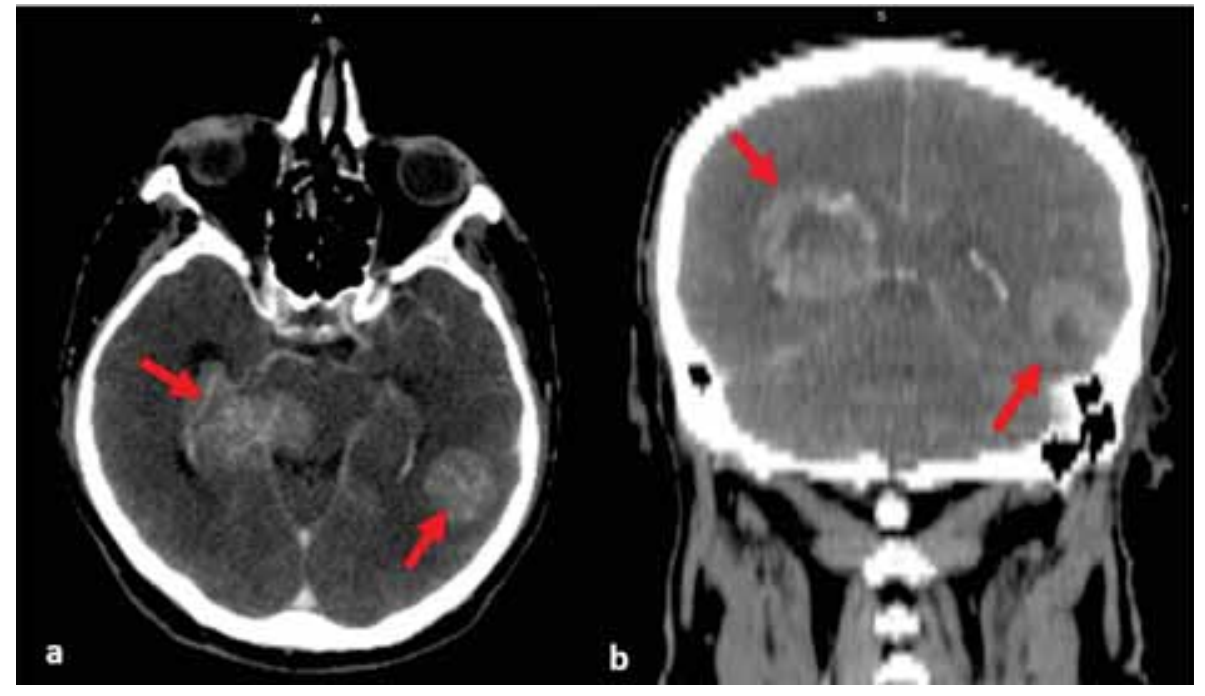

FIGURE 2. Simulation CT scan post intravenous (i.v.) contrast administration for radiotherapy planning: axial (a) and coronal (b) showing the two distinct tumors (red arrows). 


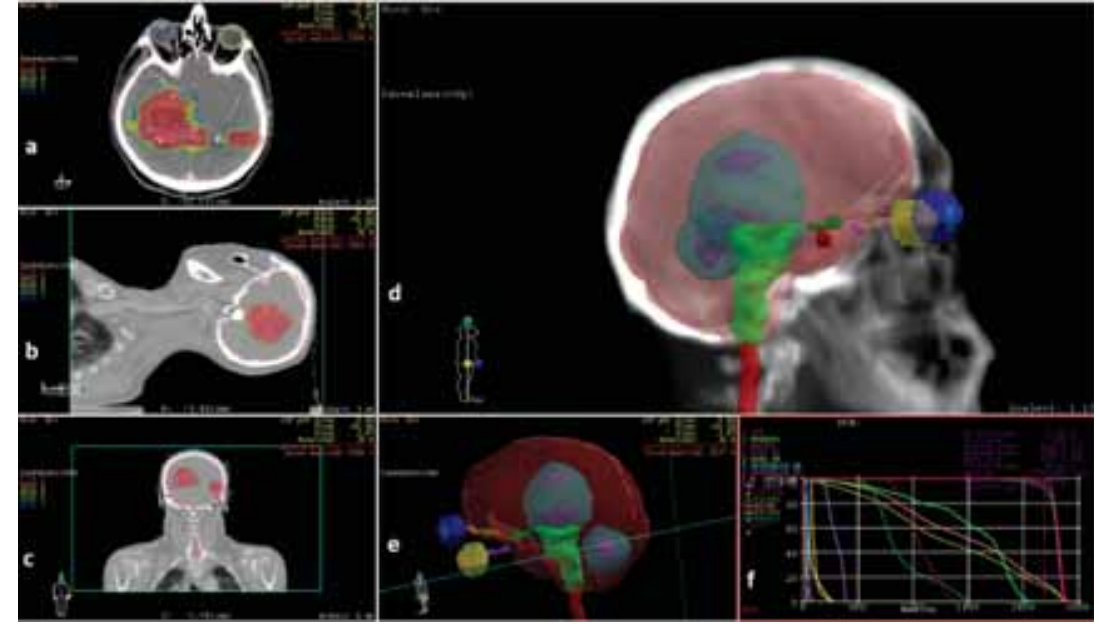

FIGURE 3. Initial radiotherapy planning: isodose distribution on axial (a), sagittal (b) and coronal (c) CT cross-sectional images; three dimensional (3D) reconstruction of $P T V$ and organs at risk (d,e); dose volume histogram (f). ium-enhancing lesions on T1-weighted MRI, clinical target volume 20 (CTV_20) as the whole brain (cerebrum, cerebellum and brainstem) and CTV_48 which encompasses the GTV plus a $2 \mathrm{~cm}$ extension with anatomical barrier editing and T2- weighted oedema regions. A $5 \mathrm{~mm}$ overlay of CTV_20 and CTV_48 was applied in order to create corresponding planning target volumes (PTV_20 and PTV_48). Brainstem, spinal cord, optic chiasma, pituitary gland, bilateral eyes, lens and optic nerves were delineated as organs at risk. Essential dosimetric parameters were analysed through dose-volume histogram (DVH) in order to comply to International Commission on Radiation Units and Measurements (ICRU) recommendation(14) (brainstem was the dose-limiting organ in this setting) (Figure 3). Radiotherapy was administered to a dose of 20 Gy in 5 fractions with 4 Gy per frac- tion to PTV_20 followed by a dose up to $48 \mathrm{~Gy}$ in 14 fractions with 2 Gy per fraction to PTV_48 with intensity modulated technique (IMRT). Considering an alpha and beta ratio of $8 \mathrm{~Gy}(15,16)$ the Equivalent Dose in 2 Gy fractions (EQD2) (17) for tumoral tissue was $52 \mathrm{~Gy}$. Combined treatment course occurred uneventfully.

Follow-up MRI evaluation revealed stable disease on the right thalamic area (Figure 4) with important necrotic tissue and moderate oedema, but a progression, according to Response Assessment in Neuro-Oncology (RANO)(18) criteria, on the left side tumor from $28 / 26 \mathrm{~mm}$ to $43 / 35 \mathrm{~mm}$. Further treatment consisted of 3 cycles of Temozolomide 150-200 $\mathrm{mg} / \mathrm{m} 2 /$ day, 5 days every 28 days and Irinotecan $125 \mathrm{mg} / \mathrm{m} 2$ days 1 and 14 .

A second hypofractionated course of volumetric modulated arc radiotherapy (VMAT) was planned,

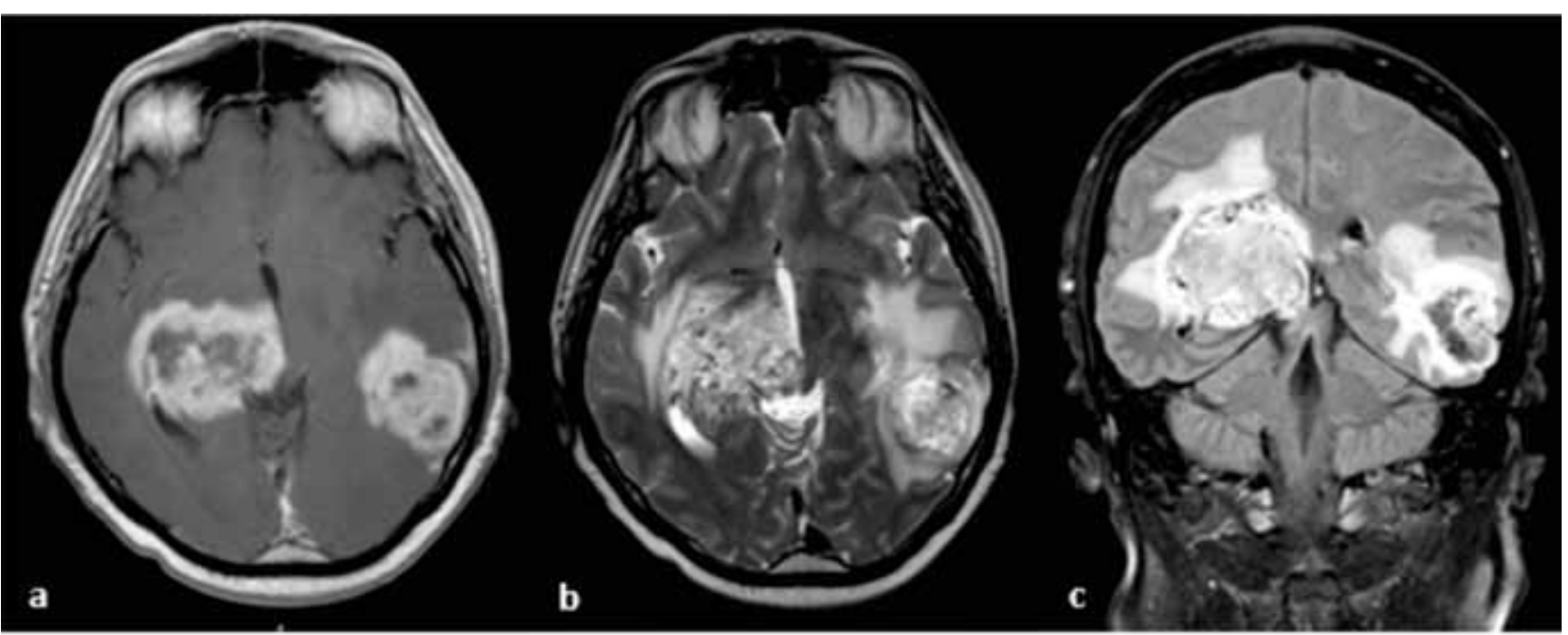

FIGURE 4. Post RT evaluation MRI: axial contrast-enhanced weighted T1 (a), axial contrast-enhanced weighted T2 (b) and coronal fluid-attenuated inversion recovery (FLAIR) (c). 


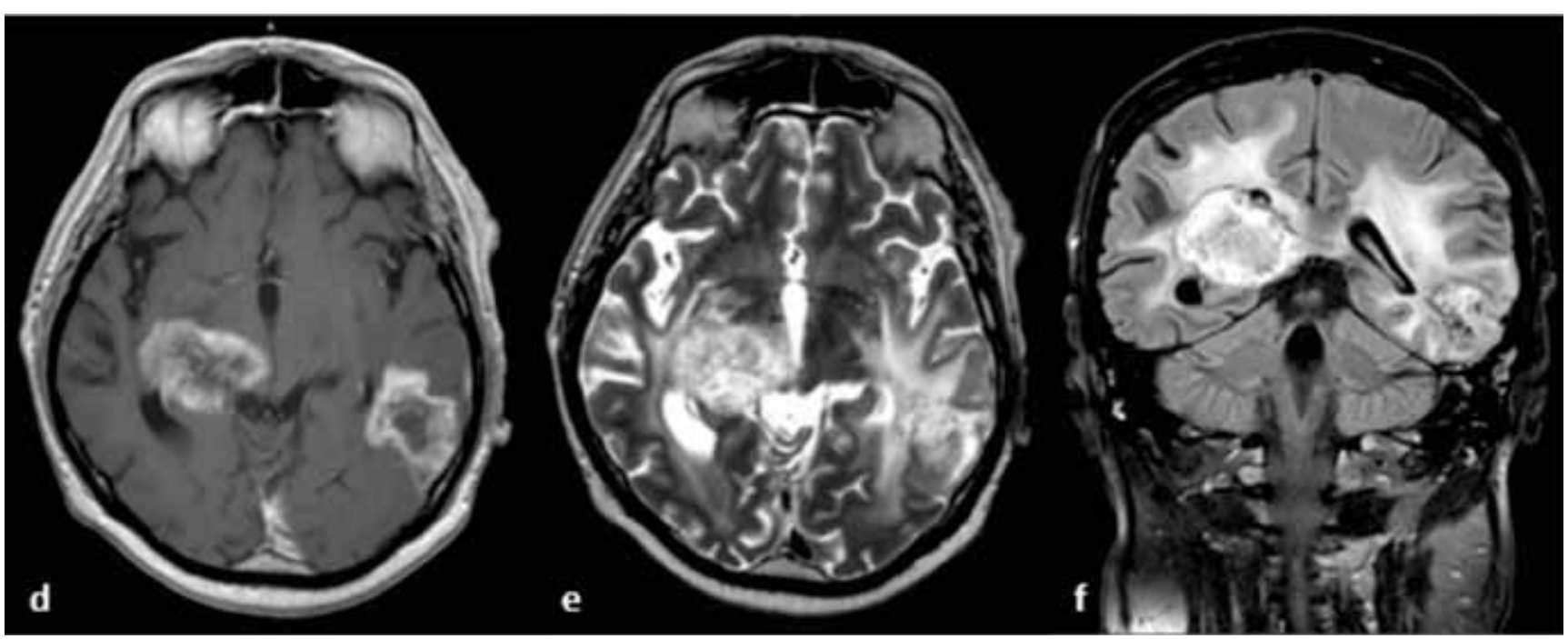

FIGURE 5. MRI follow up showing disease stabilization persistent after 11 months: axial contrast-enhanced weighted T1 (d), axial contrast-enhanced weighted T2 (e) and coronal FLAIR (f).

following the same steps as previously mentioned, to the MRI contrast-enhancing left temporal mass to a dose of 24 Gy in 6 fractions with 4 Gy per fraction (EQD2=28.8 Gy).

As the patient neurological status and general condition remained constant except for left-sided paresthesia worsening, the oncological decision was to continue monotherapy Temozolomide in the same schedule. Periodic MRIs confirmed disease stabilisation (Figure 5) for 11 months, and even if time to progression was 3 month only, the survival until present exceeds 17 months.

\section{DISCUSSION}

Glioblastomas often present as a single peripheral lesion, thus multiple enhancing masses on brain imaging are considered metastatic disease(19-21). Even if the concept of multiple gliomas, with its multicentric subtype, is a long-time characterised entity (1), the pathogenesis is indeterminate so far $(5,6,22)$. The two-phase theory was defined by Willis (23) in 1960, where neoplasia transformation of a wider brain surface is followed by separated smaller tumor sites proliferation. Hefti et al. (5) suggested that just "mirrored" lesions that have the same cell precursors are truly unlinked glioma foci, whether, in the majority of cases, unceasing distant migration is the base for distinct location tumor development.

Most of the available research data suggest that multiple glioblastomas have a poorer outcome than singular counterpart $(4,10)$, the multifocal disease being described by Syed et al. (9) as an independent prognostic factor with a negative impact on overall survival (OS) in a 265 case series, where patients with solitary tumor experience a 14.8 months OS compared to 11.5 months OS in the multifocal group. Nevertheless in cases like herein presented, longer survival intervals have been described. Peric et al. (24) reported a 19 months OS with multimodality approach combining surgery and radiochemotherapy with concomitant and adjuvant temozolomide until progression.

Several studies have shown that GBM recurrences pattern is often local $(25,26)$, limiting irradiating fields to avoid unnecessarily normal brain tissue radiation exposure, but the issue of multiple tumors still reserves a role for WBRT practice or combined approaches in patients with a poor performance status $(27,28)$. Furthermore, low-dose whole brain treatment outside standard target volume is being under investigation (29), as MRI utilization proved that tumors interconnectivity and microscopic dissemination, is more frequent than hypothesized $(3,5)$. Hypofractionated radiotherapy with different dose schedule, represent a tempting approach with a salutary treatment time reduction, as patient survival is limited, and all efforts must be made to maintain a good quality of life $(15,30)$.

\section{CONCLUSIONS}

We would like to emphasize that personalized treatment is of great importance in glioblastoma patients, even more in a particular setting like the 
multicentric presentation. The limited number of management possibilities narrows down to even fewer options beyond progression. However, above average survival intervals can be obtained with a modified multimodal approach, even in patients with poor performance status.

\section{Conflict of interest: none declared Financial support: none declared}

\section{REFERENCES}

1. Batzdorf U, Malamud N. The Problem of Multicentric Gliomas. Journal of Neurosurgery. 1963;20:122-136.

2. di Russo P, Perrini P, Pasqualetti F et al. Management and outcome of high-grade multicentric gliomas: A contemporary single-institution series and review of the literature. Acta Neurochirurgica. 2013; 155:2245-2251.

3. Lasocki A, Gaillard F, Tacey M et al. Multifocal and multicentric glioblastoma: Improved characterisation with FLAIR imaging and prognostic implications. Journal of Clinical Neuroscience. 2016; 31:92-98.

4. Thomas RP, Xu LW, Lober RM et al. The incidence and significance of multiple lesions in glioblastoma. Journal of Neuro-Oncology. 2013;112:91-97.

5. Hefti M, Campe G von, Schneider $\mathrm{C}$ et al. Multicentric Tumor Manifestations of High Grade Gliomas: Independent Proliferation or Hallmark of Extensive Disease? Central European Neurosurgery. 2010;71:20-25.

6. Salvati M, Caroli E, Orlando ER et al. Multicentric glioma: Our experience in 25 patients and critical review of the literature. Neurosurgical Review. 2003;26:275-279.

7. Hassaneen W, Levine NB, Suki D et al. Multiple craniotomies in the management of multifocal and multicentric glioblastoma. Clinical article. J. Neurosurg. 2011;114:576-584.

8. Archavlis E. Combined Salvage Therapies for Recurrent Glioblastoma Multiforme: Treatment Options in Multifocal and Multicentric Patterns of Recurrence. Journal of Cancer Prevention \& Current Research. 2017;7.

9. Syed M, Liermann J, Verma $V$ et al. Survival and recurrence patterns of multifocal glioblastoma after radiation therapy. Cancer Manag Res. 2018;10:4229-4235.

10. Patil CG, Yi A, Elramsisy A et al. Prognosis of patients with multifocal glioblastoma: A case-control study. Journal of Neurosurgery. 2012; 117:705-711.

11. Aids-to-the-Examination-of-the-Peripheral-Nervous-System-Mrc-Memorandum-No-45-Superseding-War-Memorandum-No-7.pdf. https:// $\mathrm{mrc}$.ukri.org/documents/pdf/aids-to-the-examination-of-the-peripheral-nervous-system-mrc-memorandum-no-45-superseding-war-memorandum-no-7l. Accessed November 23, 2018.

12. Oken MM, Creech RH, Tormey DC et al. Toxicity and response criteria of the Eastern Cooperative Oncology Group. Am. J. Clin. Oncol. 1982; 5:649-655.

13. Stupp R, Mason WP, van den Bent MJ et al. Radiotherapy plus concomitant and adjuvant temozolomide for glioblastoma. N. Engl. J. Med. 2005; 352:987-996.

14. Marks LB, Yorke ED, Jackson A et al. The Use of Normal Tissue Complication Probability (NTCP) Models in the Clinic. Int J Radiat Oncol Biol Phys. 2010; 76:S10-S19.

15. Hingorani M, Colley WP, Dixit S et al. Hypofractionated radiotherapy for glioblastoma: strategy for poor-risk patients or hope for the future? Br J Radiol. 2012;85:e770-e781.

16. Haas-Kogan DA, Yount G, Haas M et al. p53-dependent G1 arrest and $p 53$-independent apoptosis influence the radiobiologic response of glioblastoma. Int. J. Radiat. Oncol. Biol. Phys. 1996;36:95-103.
17. Fowler JF. 21 years of Biologically Effective Dose. Br J Radiol. 2010;83:554-568.

18. Wen PY, Macdonald DR, Reardon DA et al. Updated response assessment criteria for high-grade gliomas: Response assessment in neuro-oncology working group. J. Clin. Oncol. 2010; 28:1963-1972.

19. Prather JL, Long JM, van Heertum R et al. Multicentric and isolated multifocal glioblastoma multiforme simulating metastatic disease. BJR. 1975;48:10-15.

20. Walter J, Koch A, Herbold C et al. Multifocal glioblastoma multiforme in the posterior fossa mimicking cerebral metastases: Case presentation and review of the current literature. J Neurol Surg A Cent Eur Neurosurg. 2013;74 Suppl 1:e30-35.

21. Gasco J, Franklin B, Fuller GN et al. Multifocal epithelioid glioblastoma mimicking cerebral metastasis: Case report. Neurocirugia (Astur). 2009;20:550-554.

22. Li Z, Tian Y, Hu G et al. Multiple gliomas. Chinese Journal of Clinical Oncology. 2007;4:379-383.

23. Willis RA, Pathology of Tumours. London: Butterworth \& Co 1960: 811.

24. Peric P, Pavlicevic G, Ostojic J et al. Synchronous malignant multicentric cerebral glioma with atypical neuroradiological presentation and comparatively long survival: Case report and literature review. Vojnosanitetski pregled. 2018;75:414-421.

25. McDonald MW, Shu H-KG, Curran WJ et al. Pattern of failure after limited margin radiotherapy and temozolomide for glioblastoma. Int. J. Radiat. Oncol. Biol. Phys. 2011;79:130-136.

26. Minniti G, Amelio D, Amichetti M et al. Patterns of failure and comparison of different target volume delineations in patients with glioblastoma treated with conformal radiotherapy plus concomitant and adjuvant temozolomide. Radiother Oncol. 2010;97:377-381.

27. Showalter TN, Andrel J, Andrews DW et al. Multifocal Glioblastoma Multiforme: Prognostic Factors and Patterns of Progression. International Journal of Radiation Oncology ${ }^{*}$ Biology ${ }^{*}$ Physics. 2007;69:820-824.

28. Ampil F, Burton GV, Gonzalez-Toledo E et al. Do we need whole brain irradiation in multifocal or multicentric high-grade cerebral gliomas? Review of cases and the literature. Journal of Neuro-Oncology. 2007;85:353-355.

29. Phase II Trial of Low-Dose Whole Brain Radiotherapy With Concurrent Temozolomide and Adjuvant Temozolomide in Patients With Newly-Diagnosed Glioblastoma Multiforme - Full Text View - ClinicalTrials.gov. https://clinicaltrials.gov/ct2/show/NCT01822275. Accessed November 16, 2018.

30. Panet-Raymond V, Souhami L, Roberge D et al. Accelerated hypofractionated intensity-modulated radiotherapy with concurrent and adjuvant temozolomide for patients with glioblastoma multiforme: a safety and efficacy analysis. Int. J. Radiat. Oncol. Biol. Phys. 2009;73:473-478. 